\title{
The first detection of weak gravitational shear in infrared observations: Abell 1689
}

\author{
L. J. King ${ }^{1,2}$, D. I. Clowe ${ }^{1}$, C. Lidman ${ }^{3}$, P. Schneider ${ }^{1,2}$, T. Erben ${ }^{1,4,5}$, J.-P. Kneib ${ }^{6}$, and G. Meylan ${ }^{7}$ \\ 1 Institut für Astrophysik und Extraterrestrische Forschung, Auf dem Hügel 71, 53121 Bonn, Germany \\ 2 Max-Planck-Institut für Astrophysik, Karl-Schwarzschild-Strasse 1, 85741 Garching, Germany \\ 3 European Southern Observatory, Casilla 19, Santiago, Chile \\ 4 Institut d'Astrophysique de Paris, 98 bis Boulevard Arago, 75014 Paris, France \\ 5 Observatoire de Paris, DEMIRM, 61 avenue de l'Observatoire, 75014 Paris, France \\ 6 Observatoire Midi-Pyrénées, 14 Av. E. Belin, 31400 Toulouse, France \\ 7 Space Telescope Science Institute, 3700 San Martin Drive, Baltimore, MD 21218, USA
}

Received 14 January 2002 / Accepted 13 February 2002

\begin{abstract}
We present the first detection of weak gravitational shear at infrared wavelengths, using observations of the lensing cluster Abell 1689, taken with the SofI camera on the ESO-NTT telescope. The imprint of cluster lenses on the shapes of the background galaxy population has previously been harnessed at optical wavelengths, and this gravitational shear signal enables cluster mass distributions to be probed, independent of whether the matter is luminous or dark. At near-infrared wavelengths, the spectrophotometric properties of galaxies facilitate a clean selection of background objects for use in the lensing analysis. A finite-field mass reconstruction and application of the aperture mass $\left(M_{\mathrm{ap}}\right)$ statistic are presented. The probability that the peak of the $M_{\text {ap }} \operatorname{detection}\left(\frac{S}{N} \sim 5\right)$, arises from a chance alignment of background sources is only $\sim 4.5 \times 10^{-7}$. The velocity dispersion of the best-fit singular isothermal sphere model for the cluster is $\sigma_{1 \mathrm{D}}=1030_{-80}^{+70} \mathrm{~km} \mathrm{~s}^{-1}$, and we find a $K$-band mass-to-light ratio of $\sim 40 M_{\odot} / L_{\odot}$ inside a $0.44 \mathrm{Mpc}$ radius.
\end{abstract}

Key words. gravitational lensing - galaxies: clusters individual: Abell 1689 - dark matter - infrared: galaxies

\section{Introduction}

A cluster acting as a weak gravitational lens distorts the shapes of background galaxies by virtue of its tidal gravitational field. The seminal paper of Kaiser \& Squires (1993) describes how to use this information to obtain a parameter-free reconstruction of a mass distribution. Their technique, and variants thereof, has been applied to optical observations of a number of clusters (e.g. Clowe et al. 2000; Hoekstra et al. 2000).

Prior to this work, there has been no published report on the detection of gravitational shear in infrared observations. For a given integration time, the attainable number density of background galaxies for use in weak lensing studies is usually much lower than for optical observations. In addition, infrared detectors currently lag behind optical detectors in their field-of-view making them even less efficient for wide-field studies. Nevertheless, with developments in detector technology, and new widefield infrared cameras becoming available, this is an avenue to be explored. Gray et al. (2000) have considered

Send offprint requests to: L. J. King, e-mail: lindsay@astro.uni-bonn.de the magnification induced depletion effect in near-infrared CIRSI observations of the cluster Abell 2219, in order to obtain best-fit SIS (singular isothermal sphere) and NFW (Navarro et al. 1996) models.

Here we report on $J, H$ and $K$ s infrared observations in which we detect the gravitational shear signature of the cluster Abell $1689(z=0.182)$, manifest in the distorted images of background galaxies. We start by outlining the observations, and the strategy for obtaining a catalogue of background galaxies for use in the weak lensing analysis. Then we describe how this catalogue was used to perform a mass reconstruction of the cluster. The $M_{\text {ap }}$ statistic of Schneider (1996) is then calculated, along with a quantitative measure of its significance. Next we use $K$ s selected cluster members to determine the luminosity of the lens, and obtain its mass-to-light ratio by comparison with the mass derived from fitting an SIS profile to the weak lensing data. We conclude with a brief discussion of the results, and the implications for future work. For quantitative estimates, we assume a cosmology with $\Omega=0.3, \Lambda=0.7$ and $H_{0}=70 \mathrm{~km} \mathrm{~s}^{-1} \mathrm{Mpc}^{-1}$, and that the background galaxy population is at $z=1.0$. The conversion between angular and linear scales at $z=0.182$ is $1^{\prime} .0 \equiv 0.18 \mathrm{Mpc}$. 
Table 1. The integration time $(t)$, image quality $(I Q)$, sensitivity limit $(S L)$ and extinction $(A)$ are shown for each of the filters with which A1689 was observed.

\begin{tabular}{lcccc}
\hline Filter & $t(\mathrm{ks})$ & $I Q\left(^{\prime \prime}\right)$ & $S L(\mathrm{mag})$ & $A$ (mag/airmass) \\
\hline$J$ & 14.4 & 0.77 & 25.59 & 0.07 \\
$H$ & 10.8 & 0.85 & 24.21 & 0.04 \\
$K \mathrm{~s}$ & 15.6 & 0.73 & 23.57 & 0.07 \\
\hline
\end{tabular}

\section{Observations and data reduction}

Our data were obtained on the nights 2000 April 29th and 30th, using the SofI camera (Moorwood et al. 1998) on the ESO-NTT telescope at Cerro La Silla Observatory, Chile. The instrument was used in "Large Field Imaging" mode, where the field of view is $4^{\prime} .9$ on a side, and the pixel scale is 0 ' 29 /pixel. Abell 1689 was observed in three filters, $J$, $H$ and $K$ s. Individual integrations lasted between 10 and $30 \mathrm{~s}$ and several of these were co-added to form an individual image. Between images, the telescope was moved by up to 2 '.0, while always keeping the target within the field of view. This offset is somewhat larger than is typical in such observations; however, it was necessary given the high density of galaxies in the centre of A1689. The field of view is effectively increased to $\sim 7^{\prime} .0$ (1440 pixels). During the observations the seeing was typically $\sim 0$.' 8 and conditions were perfectly photometric, except during the early hours of the first night.

The data were reduced in the standard way. From each image, the zero-level offset was removed, the flat field correction was applied, and then an estimate of the sky from other images in the sequence was subtracted. The images were combined so that the $\frac{S}{N}$ (signal-to-noise) ratio of the final image was maximised and with linear registration relative to the first frame in each sequence.

The photometric calibration was done by observing several stars from the list of HST NICMOS photometric standards (Persson et al. 1998). Although there was some thin cirrus early in the first night, we were able to observe a sufficiently large number of standards to derive accurate instrumental zero points and atmospheric extinction coefficients in each of the three filters. The total integration times $(t)$, the final image quality $(I Q)$, the sensitivity limit (1- $\sigma$ noise limit in 1 square arcsecond) ( $S L)$ and extinction coefficients $(A)$ of the combined images in the $J, H$ and $K \mathrm{~s}$ bands are given in Table 1 . The instrumental zero points were very stable over the two nights and the scatter in the fit of the instrumental magnitude versus airmass is less than $0.01 \mathrm{mag}$ in all three filters.

We did not correct for colour terms between the SofI instrumental system and that used for the HST NICMOS standards. Preliminary estimates suggest that these terms are all less than 0.02 mag. Nor did we correct the flat fields for illumination effects which cause the photometry to vary differentially across the field by up to $0.03 \mathrm{mag}$. Thus, the accuracy of the relative and absolute photometry is around 0.03 mag for both.

\section{Obtaining a catalogue of background objects}

The goal described in this section is the acquisition of a catalogue of background object positions and ellipticities, that can be used in the weak lensing analysis.

Our strategy was to start by using SExtractor (Bertin \& Arnouts 1996) to detect objects on each of the $J, H$ and $K$ s images separately. For all filters, an object detection and analysis threshold of at least 3 contiguous pixels above $1 \sigma$ was required. Since the $J$ image contained most detections, the $H$ and $K$ s images were transformed to the $J$ reference frame, using IRAF tasks (see Tody 1993 and references therein). SExtractor was then run in "doubleimage mode", using the $J$ image for detection (with an object detection and analysis threshold of at least 2 contiguous pixels above $0.7 \sigma$ ) and each of the $J, H$ and $K \mathrm{~s} \mathrm{im-}$ ages for measurements. For each object, magnitudes were measured using both a 15 pixel (4". 4) diameter aperture, and an outer isophote at $70 \%$ of the sky noise $(J: 24.07$, $H: 23.11, K \mathrm{~s}: 22.55 \mathrm{mag} / \mathrm{sq}$ arcsec). Aperture magnitudes are used for colour determination, and isophotal magnitudes are used for all other purposes. The LDAC tools ${ }^{1}$ were used to obtain a single catalogue of these sources.

Ellipticities for the objects were determined from the second moments of their surface brightness using a modified version of Nick Kaiser's IMCAT ${ }^{2}$ software. Bright (but unsaturated) stars were selected on the basis of their halflight radii and were used to determine the point spread function (PSF) as a function of position in the images. A bi-cubic polynomial was fit to the stellar ellipticities (for 25 stars) and used to correct the ellipticities of the galaxies using the technique detailed in Kaiser et al. (1995). The smearing of the galaxy ellipticities by the now circular PSF was removed using the basic method outlined in Luppino \& Kaiser (1997), but where the pre-seeing shear polarizability tensor was fit as a 5th order polynomial function of $r_{\mathrm{g}}$, the Gaussian smoothing radius at which the galaxy achieves maximum significance from the background, and the post-seeing ellipticity. The resulting shear estimate has been shown to be good to a few percent accuracy for well behaved PSFs such as those in these images (Erben et al. 2001).

Stellar objects were filtered out, based on their locus in a magnitude-radius plot. Next, making use of the small dispersion in colour at a particular magnitude for cluster galaxies, likely cluster members were excised to exclude even the faintest cluster members, where the dispersion in the cluster sequence becomes larger.

Further, only galaxies fainter than $m_{J}=16.6$ and having a $\frac{S}{N}>5$ in $J$ were retained. The $\frac{S}{N}$ limit corresponds to $m_{J}=23.2$, for the smallest objects of similar size to the PSF. Finally, only galaxies redder than the cluster sequence galaxies were kept in the catalogue (they are most probably at higher redshifts than the cluster; see for example the colour evolution plots in Poggianti 1997).

\footnotetext{
${ }^{1}$ Freeware available from ftp://ftp.strw.leidenuniv.nl/ pub/ldac/

${ }^{2}$ http://www.ifa.hawaii.edu/ kaiser/imcat
} 


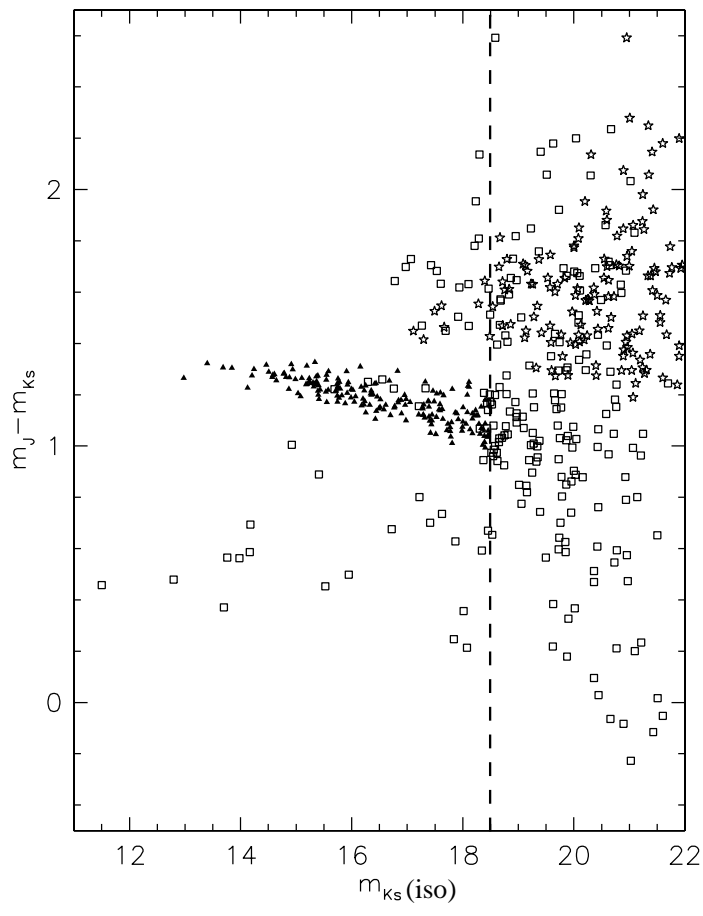

Fig. 1. The colour-magnitude diagram for objects selected as background galaxies for use in the lensing analysis (open stars). The dashed line at $m_{K \mathrm{~s}}=18.5$ indicates the limit down to which objects selected as cluster galaxies were used in the luminosity estimation (solid triangles). Other objects detected but not used in the lensing or mass-to-light analysis, including stars, fainter cluster members, and cluster members outside the radius inside which the mass-to-light ratio is determined are marked with open squares. Here, $m_{K \mathrm{~s}}$ and $m_{J}$ are aperture magnitudes, and $m_{K \mathrm{~s}}($ iso $)$ is an isophotal magnitude. Note the clear colour-magnitude sequence of cluster galaxies.

The selection process for the catalogue as outlined above is the most conservative of our trials, and the final number of sources used in the weak lensing analysis is 157 , just over $3 / \operatorname{arcmin}^{2}$. In Fig. 1 , these objects are indicated (open stars) on a colour-magnitude diagram for the observations. We experimented with various cuts, which induce only a marginal change in the results of the lensing analysis described in the next section.

\section{Weak lensing analysis}

For the weak lensing analysis, we used the mean of the shear estimates determined from the separate $J, H$ and $K$ s images, weighted by the $\frac{S}{N}$ of the object detections. The best-fit SIS model to the radial shear profile (centred on the brightest cluster galaxy) has a velocity dispersion $\sigma_{1 \mathrm{D}}=1030_{-80}^{+70} \mathrm{~km} \mathrm{~s}^{-1}$, with a significance of 6.1 relative to the null model $\left(\sigma_{1 \mathrm{D}}=0 \mathrm{~km} \mathrm{~s}^{-1}\right)$. This value is consistent with the best-fit SIS to WFI optical data $\left(\sigma_{1 \mathrm{D}}=998_{-42}^{+33} \mathrm{kms}^{-1}\right)$ presented in King et al. (2002). The best-fit NFW model has $r_{200}=1.94 \mathrm{Mpc}$ and $c=5.7$, the significance and quality of fit being the same as the SIS. We checked that fitting a radial shear profile to the objects bluer than the cluster sequence results in an insignificant weak lensing signal.

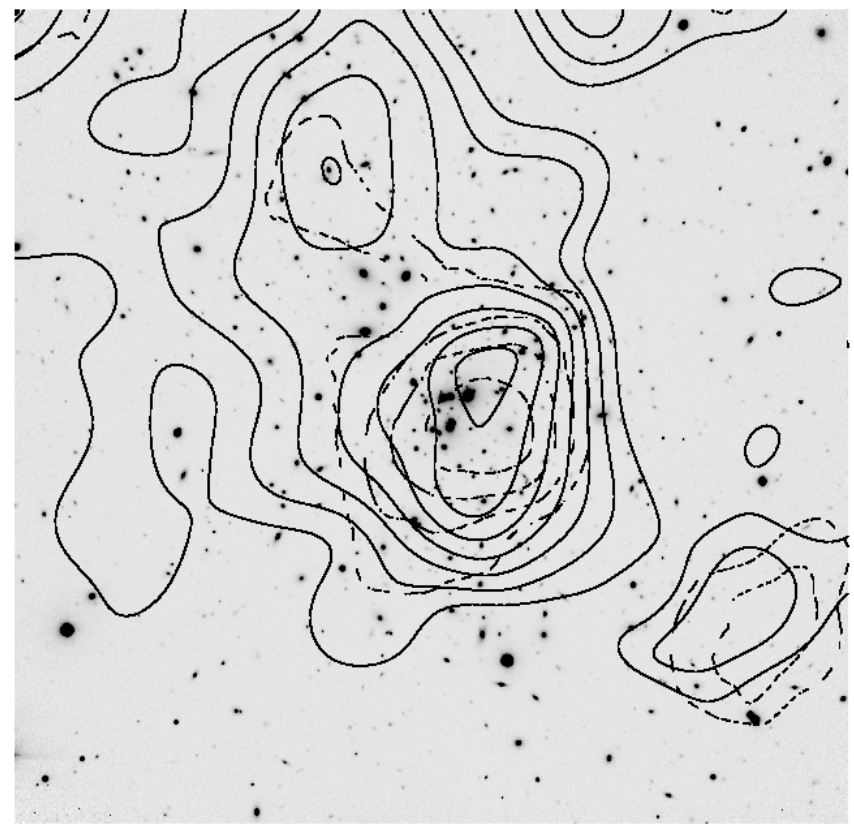

Fig. 2. The greyscale shows the SofI J-band image, 7.0 on a side, $\mathrm{N}$ to the top and $\mathrm{E}$ to the left. The solid contours show the surface mass density $\kappa$ from a finite-field mass reconstruction with a smoothing scale of 0.54 , plotted at intervals of 0.05 in $\kappa$ relative to the boundary value. A contour plot of $\frac{S}{N}$ for $M_{\mathrm{ap}}$ with a filter scale of $\eta=1^{\prime} .95$ is plotted as dashed contours, corresponding to $\frac{S}{N}=1,2,3,4$.

\subsection{Mass reconstruction}

We use the non-parametric finite-field mass reconstruction algorithm detailed in Seitz \& Schneider (2001). The reconstruction was performed with a smoothing scale of $0^{\prime} .54$. The result is shown as solid contours in Fig. 2, normalised (necessary because of the mass-sheet degeneracy) so that the mean surface mass density on a $0^{\prime} .097$ wide border is $\bar{\kappa}=0.1$. Note that the mass peak is consistent with that of the luminous infrared image.

Since the noise properties for non-parametric reconstructions are not easily interpreted, multiple reconstructions were performed during which the catalogue object positions were kept fixed and ellipticities were assigned at random (with replacement) from the ellipticity ensemble. This enabled us to get a handle on the noise properties, and confirms that the central mass concentration is not a noise artefact.

\subsection{Aperture mass statistic}

Next we consider the $M_{\mathrm{ap}}$ statistic, which measures the surface mass density convolved with a compensated Mexican Hat filter function $\mathcal{U}$. Equivalently, the suitably weighted ellipticities give a practical estimator of $M_{\mathrm{ap}}$

$M_{\mathrm{ap}}(\boldsymbol{\vartheta})=\frac{\pi \eta^{2}}{N} \sum_{i} \epsilon_{\mathrm{ti}}(\boldsymbol{\vartheta}) \mathcal{Q}\left(\left|\boldsymbol{\theta}_{i}-\boldsymbol{\vartheta}\right|\right)$,

where the sum extends over $N$ galaxies, located at positions $\boldsymbol{\theta}_{i}$, inside a (filter) radius $\eta$ centred on position $\boldsymbol{\vartheta}$. 
The quantity $\epsilon_{\mathrm{t}}(\boldsymbol{\vartheta})$ is the tangential component of the ellipticity with respect to position $\vartheta$, and $\mathcal{Q}$ is a filter function related to $\mathcal{U}$. We use the simplest form of the filter functions from Schneider et al. (1998).

An asset of this statistic is that the noise properties are well understood, and the significance of a $M_{\text {ap }}$ detection is readily calculated by comparing its value with that obtained from the same set of galaxies with random position angles. The signal to noise ratio $\frac{S}{N}$ for $M_{\text {ap }}(\boldsymbol{\vartheta})$ is $\frac{M_{\mathrm{ap}}}{\sqrt{\sigma^{2}}}(\boldsymbol{\vartheta})$ where $\sigma^{2}=\left\langle M_{\mathrm{ap}}^{2}\right\rangle_{\text {randomisations }}(\boldsymbol{\vartheta})$. The rms dispersion of $M_{\mathrm{ap}}$ is calculated in the absence of lensing, since the deviation from this approximation is very small in the weak lensing regime; it gives an upper limit on $\sigma$. The $\frac{S}{N}$ of $M_{\text {ap }}$ calculated for a filter scale $\eta=1.95$ and for 1000 randomisations, is shown as dashed contours in Fig. 2. $\frac{S}{N} \sim 5$ for the peak of the detection; its location is consistent with the peak of the non-parametric mass reconstruction and with the brightest cluster galaxy on the infrared image. Also, note that the $M_{\text {ap }}$ detection is highly significant over a large area.

The calculation of $\frac{S}{N}$ in the previous paragraph relies on the fact that $M_{\text {ap }}(\boldsymbol{\vartheta})$ has a Gaussian probability distribution. In addition, $M_{\text {ap }}$ was calculated around the peak of the detection for $4 \times 10^{7}$ randomisations of the background galaxy orientations, and a higher $\frac{S}{N}$ peak was obtained for only 18 of these cases. The probability that such a random distribution of galaxies would give a $\frac{S}{N}$ in excess of the observed peak value is therefore $\sim 4.5 \times 10^{-7}$. As another check, the position angles of the background galaxies were rotated through $45^{\circ}$, and no highly significant $M_{\text {ap }}$ detection was obtained.

\section{Mass-to-light ratio}

Selection of cluster members was performed in the $K_{\mathrm{s}}$ band data, down to isophotal $m_{K \mathrm{~s}}=18.5$ and using their tight colour-magnitude and colour-colour loci. Further, we restricted selection to objects within 500 pixels $(2 ' 43)$ of the brightest cluster galaxy. Outside of this radius, the sky noise increases dramatically due to dithering causing a decrease in exposure time. We indicate the objects selected as cluster members in Fig. 1. The apparent magnitudes of these galaxies were converted to absolute magnitudes, and subsequently to luminosities using the evolutionary and $\mathrm{k}$-corrections obtained from the single burst, solar metallicity, Scalo (1986) IMF spectral energy distribution contained in HyperZ (Bolzonella et al. 2000) (amounting to $-0.53 \mathrm{mag})$ and the extinction correction from our data (0.09 mag).

The differential $K$-band luminosity function of the cluster members is shown in Fig. 3, with error bars corresponding to the Poisson variance in each bin, along with the best-fit Schechter (1976) luminosity function

$$
\Phi(L) \mathrm{d} L=\Phi^{*}\left(\frac{L}{L_{*}}\right)^{\alpha} \exp \left(-\frac{L}{L_{*}}\right) \frac{\mathrm{d} L}{L_{*}} .
$$

The nonlinear least-squares Marquardt-Levenberg algorithm was used to obtain the best-fit parameters for the

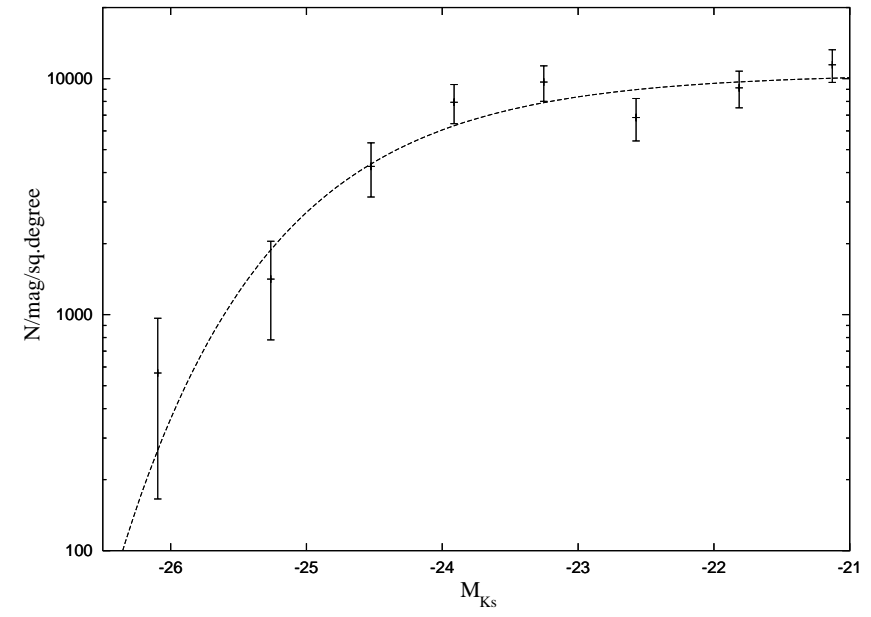

Fig. 3. The luminosity function of the $K$ s selected cluster galaxies, with the best-fit Schechter function indicated by the dashed line. Error bars correspond to Poisson noise in each bin.

faint slope $\alpha=-1.01 \pm 0.14$ and characteristic absolute magnitude $M_{*}=-24.7 \pm 0.40$ (i.e. $m_{*}=14.6$ ), with a reduced $\chi^{2}$ of 1.3. Taking into account the difference in adopted cosmology, this value of $M_{*}$ is very similar to that obtained by Barger et al. (1996), for a sample of distant clusters.

The integrated luminosity (extrapolated to zero luminosity) for the best-fit Schechter function is $8.0 \times 10^{12} L_{\odot}$ and the direct sum of galaxy luminosities (down to $L_{\text {min }}$ ) is $8.8 \times 10^{12} L_{\odot}$. These are consistent, given the errors of the fit parameters. We use the direct sum in the mass-tolight ratio estimate, since we reach well down the luminosity function: the fraction of light observed is approximately $\Gamma\left(\alpha+2, \frac{L_{\min }}{L_{*}}\right) / \Gamma(\alpha+2) \sim 97 \%$. In order to estimate the error on the luminosity, first consider the error arising from the various cuts in the colour-magnitude and colourcolour planes, given that each object has a photometric uncertainty. We started with the original uncut catalogue, and created 100000 mock catalogues, assigning the $i$ th object a flux $F_{i}=F_{i \text { (mea) }}+g F_{i(\text { err })}$, where $g$ is a uniform Gaussian random variable, and $F_{i \text { (mea) }}, F_{i \text { (err) }}$ are the measured flux and its error, respectively. The magnitude and colour cuts were then applied to these catalogues, and the dispersion in the recalculated luminosity determined to be only $\sim 0.35 \%$ of the total. Another very small error is the Poissonian error on the total flux, which amounts to $\sim 0.05 \%$. The dominant error is the $2 \%$ error on the luminosity because of uncertainty in the $K$ s zero point. Hence for the cluster luminosity we take $8.8 \pm 0.18 \times 10^{12} L_{\odot}$ (which is a lower limit because $\sim 3 \%$ of the luminosity is expected to be below the $K \mathrm{~s}$ magnitude limit, and light outside the isophotal radii of galaxies is not accounted for).

The projected mass of the best-fit SIS to the infrared data within the same $2^{\prime} .43$ radius is $M=3.44_{-0.51}^{+0.49} \times$ $10^{14} M_{\odot}$. This gives an upper-limit $K$-band mass-to-light ratio of $39.1 \pm 5.7 M_{\odot} / L_{\odot}$. The best-fit NFW model has a mass at this radius which is $\sim 10 \%$ higher. 


\section{Discussion and conclusions}

We find that it is possible to fit a radial shear profile (the best-fit SIS model has $\sigma_{1 \mathrm{D}}=1030_{-80}^{+70} \mathrm{~km} \mathrm{~s}^{-1}$ ), perform a mass reconstruction, and obtain a $M_{\text {ap }} \frac{S}{N} \sim 5$ detection using only the infrared data on a relatively small field. The locations of the peaks of the reconstruction and of the $M_{\text {ap }}$ detection are consistent with that of the brightest cluster galaxy in the infrared image.

The $K$-band luminosity function of the cluster galaxies is well described by a Schechter function, with a faint end slope $\alpha=-1.01 \pm 0.14$ and characteristic absolute magnitude $M_{*}=-24.7 \pm 0.40$. In addition, we find a $K$-band mass-to-light ratio of $\sim 40 M_{\odot} / L_{\odot}$ in the cosmology adopted, in accord with the study of rich Abell clusters made by Uson \& Boughn (1991).

The capability to perform routine wide-field infrared imaging is just around the corner with projects such as the UKIRT WFCAM, the CFHT WIRCAM and VISTA. Weak gravitational lensing observations of distorted galaxies in the infrared will provide another window through which to probe the mass distribution on cluster scales. Although the number density of background sources achievable is roughly an order of magnitude lower at infrared wavelengths, there are a few points worth noting:

- Colours of galaxies in the near-infrared are dominated by light from old evolved stars, so the well defined cluster sequence in the colour-magnitude diagram contains both ellipticals and (unlike the optical cluster sequence) spirals. This makes excising potential cluster members easier using infrared colours, as anything redder than the sequence is likely to be at a higher redshift, and anything bluer is probably at a lower redshift. The infrared selection of background objects can then be used as a consistency check of the optical selection, to assess the contamination from cluster members and foreground objects.

- With optical observations, measurement of the ellipticities of high redshift galaxies can be hampered by the presence of blue knots of star formation.

- The total $K$-band luminosity of the cluster members can be compared with a weak lensing mass, in order to obtain a mass-to-light ratio. Further, Broadhurst et al. (1992) underlined that the $K$-band luminosity is a good measure of the underlying stellar mass irrespective of how it assembled. If a high enough resolution mass map became available, we could investigate the correlation between any peaks in the total mass and stellar mass content.

Although infrared observations are not preferable to optical ones if the goal is simply to do a lensing analysis, this work highlights the complementary nature of this passband. Our next step is to combine the infrared and optical information. On a galaxy-by-galaxy basis, the selection of background galaxies can be examined. In addition, we can compare the ellipticities of the lensed galaxies determined from each data set. Finally, we will consider constraints from strong lensing features to obtain the most precise description of the mass distribution of this spectacular lens.

Acknowledgements. This work was supported by the TMR Network "Gravitational Lensing: New Constraints on Cosmology and the Distribution of Dark Matter" of the EC under contract No. ERBFMRX-CT97-0172, and by the DFG and the CNRS. Thanks to Joan-Marc Miralles, Roser Pelló, Tom Broadhurst, Michael Hilker, Martin Altmann and Neil Trentham for useful discussions, and to Leonardo Vanzi for providing us with flat field images from the SofI calibration plan. Thanks also to the referee for very helpful comments on the manuscript.

\section{References}

Barger, A. J., Aragon-Salamanca, A., Ellis, R. S., et al. 1996, MNRAS, 279, 1

Bertin, E., \& Arnouts, S. 1996, A\&AS, 117, 393

Bolzonella, M., Miralles, J.-M., \& Pelló, R. 2000, A\&A, 363, 476

Broadhurst, T. J., Ellis, R. S., \& Glazebrook, K. 1992, Nature, 355,55

Clowe, D., Luppino, G. A., Kaiser, N., \& Gioia, I. M. 2000, ApJ, 539, 540

Clowe, D., \& Schneider, P. 2001, A\&A, 379, 384

Erben, T., Van Waerbeke, L., Bertin, E., Mellier, Y., \& Schneider, P. 2001, A\&A, 366, 717

Gray, M. E., Ellis, R. S., Refregier, A., et al. 2000, MNRAS, 318,573

Hoekstra, H., Franx, M., \& Kuijken, K. 2000, ApJ, 532, 88

Kaiser, N., \& Squires, G. 1993, ApJ, 404, 441

Kaiser, N., Squires, G., \& Broadhurst, T. 1995, ApJ, 449, 460

King, L. J., Clowe, D. I., \& Schneider, P. 2002, A\&A, 383, 118

Luppino, G., \& Kaiser, N. 1997, ApJ, 475, 20

Morwood, A., Cuby, J.-G., \& Lidman, C. 1998, ESO Messenger, 91, 9

Navarro, J. F., Frenk, C. S., \& White, S. D. M. 1996, ApJ, 462, 563

Persson, S. E., Murphy, D. C., Krzeminski, W., Roth, M., \& Rieke, M. J. 1998, AJ, 116, 2475

Poggianti, B. M. 1997, A\&AS, 122, 399

Scalo, J. M. 1986, FCPh, 11, 1

Schechter, P. 1976, ApJ, 203, 297

Schneider, P. 1996, MNRAS, 283, 837

Schneider, P., van Waerbeke, L., Jain, B., \& Kruse, G. 1998, MNRAS, 296, 873

Seitz, S., \& Schneider, P. 2001, A\&A, 374, 740

Tody, D. 1993, ASP Conf. Ser., 52, 173, ed. R. J. Hanisch, R. J. V. Brissenden, \& J. Barnes

Uson, J. M., \& Boughn, S. P. 1991, ApJ, 369, 38 